\title{
Assuming the mantle: the balancing act facing the new WHO Director-General
}

\author{
Kelley Lee Kent Buse
}

J R Soc Med 2006;99:494-496

This is the first in a series of articles on leadership in international health.

In November 2006 a new Director-General of the World Health Organisation (WHO) will be elected to replace Lee Jong-wook who died earlier this year. The process for selecting the new leader, which began in early summer, has invariably been a highly political one. The job offers the incumbent the opportunity to influence global health at the highest level but it is also a heavily demanding role requiring skilful balancing of diverse needs, priorities and interests. Thus, as well as vision and sound technical expertise, the winning candidate should be armed with the skills of a diplomat, negotiator and leader.

The first challenge facing the new Director-General is deciding where WHO should target its efforts given unlimited health needs, limited resources and a plethora of other global and international health agencies. The organization has been accused in the past of trying to do too much, spreading itself thinly by failing to identify clear priorities. In part, this inclusive approach to programme activity stems from the broad concept of health set out in its 1948 Constitution. Debates on priority setting have raged ever since. How do you practically convert the aspiration to achieve 'the highest attainable standard of health', with health defined as 'a state of complete physical, mental and social well-being and not merely the absence of disease or infirmity', into an effective programme of work?

The process of setting the Eleventh General Programme of Work, ${ }^{1}$ covering the ten-year period from 2006-2015, has reflected this ongoing challenge. The programme is intended to provide 'a long term perspective on determinants and trends in health' and a broad strategic framework to guide the work of member states, partner organizations and the WHO secretariat. The draft programme, however, immediately met with concerns among Executive Board members that it focused on the global health agenda rather than what WHO would

London School of Hygiene \& Tropical Medicine, Keppel Street, London WC1E 7HT; Overseas Development Institute, 111 Westminster Bridge Road, London SE1 7JD

e-mail: K.buse@odi.rg.uk be doing. A major rewrite of the Programme was undertaken earlier this year, along with an effort to establish clearer priorities by reducing the number of priority areas set out in the budget for the medium-term strategic plan (MTSP) from 36 in 2006-2007 to 16 for 2008-2009. This is accompanied by a more robust set of indicators, agreed by an external group of health measurement and statistics experts, to monitor the achievement of objectives. In this way, it is believed that the plan will 'enable WHO to respond in a flexible and dynamic manner to a changing international health environment.' 2

WHO's modest budget, at around US\$3300 million for 2006-2007, and where these funds come from also makes programming harder. Regular budget funds (RBFs) comprise assessed contributions from all member states over which WHO exerts discretionary control. Extrabudgetary funds (EBFs) are largely earmarked by a small number of funders (mainly bilateral agencies) for specified purposes and time periods, a practice that has increased as donors have sought more control over planning and financial management. A decade ago the amount of RBFs versus EBFs was roughly the same. ${ }^{3}$ Over time RBFs (US\$915 million in 2006) have shrunk in real terms and relative to EBFs (US\$2398 million). In principle, WHO's General Programme of Work sets out the organization's priorities. In practice, funders heavily influence what is given greatest attention, leaving RBFs to gap fill across the organization's various programme 'clusters' and six geographical regions. Even allocating RBFs internally requires the organization to engage in tricky negotiation. Efforts to reallocate RBFs across programmes and regions, and align them more closely with agreed priorities, have often met with intransigence, calling for political skills of the highest order to overcome this resistance. Recognizing this lack of room to manoeuvre, and the need for more predictability of funding, the draft medium-term strategic plan (2008-2013) calls for an increase in assessed contributions to US $\$ 1000$ million in order to maintain a 'reasonable balance' between the two sources of funding. Many funders have acknowledged these problems, along with WHO's positive efforts to reform its financial management, budgeting and management practices. Memorandums of 
understanding have been agreed with some donors for more predictable 'core' EBFs, along with increased RBFs. The new Director-General will be expected to use this growing and more stable budget effectively.

The nature of core activities that WHO should be engaged in poses the next immediate challenge. Should WHO focus on technical activities such as developing norms and standards, shaping research agendas, agreeing nomenclature, providing guidance for policy development, and collecting essential health data? Or should it be more operational, rolling up its sleeves to deliver health interventions on the ground? Some critics point to the concentration of resources at headquarters and regional offices as evidence of WHO's penchant for saying but not doing. Since the late 1990s, under Gro Harlem Brundtland and then Lee Jong-wook, efforts have been made to shift more staff and control over money to the regional and country levels for technical activities. Unfairly eclipsed at times by more media friendly organisations such as UNICEF, WHO has been enticed to take a more operational approach. The ' 3 by 5 ' initiative to laudably increase access to life-saving anti-retroviral treatment in low-income countries, announced in 2002, is a case in point. One of the five main areas agreed within the MTSP is for WHO to provide 'support to countries in moving to universal coverage with effective public-health interventions'. For the ' 3 by 5 ' initiative, some have interpreted 'support' as operational activities. Along with concerns that the approach is narrow, top-down and expensive, ${ }^{4}$ most agree that broader aspirations to be more action-oriented would threaten WHO's perceived comparative advantage, as well as strain its limited resources and capacity. A greater operational role, for example, would require WHO to recruit a large number of new staff with very different skills. Rather than emulating others, and reinventing itself as an implementing agency, it would seem more sensible for WHO to reinvigorate its reputation by more effectively fulfilling its global oversight role. ${ }^{5}$

Indeed, there is no lack of operational players in the global health arena and WHO would do well to avoid adding to the notoriously uncoordinated scrum on the ground. Asserting its place amid the crowd of institutions jostling for space will need to be tackled by the new Director-General. WHO's leadership role as the United Nations specialized agency for health has undoubtedly been diluted by the World Bank's entry into the sector; the creation of the UN Programme on HIV/AIDS (UNAIDS); the Global Fund to Fight HIV/AIDS, Tuberculosis and Malaria; and other health initiatives outside of its auspices. ${ }^{6}$ The growth of private philanthropy in recent years, led by the flushed coffers of the Bill and Melinda Gates Foundation, might also be interpreted as a rival to the crown. Yet, in tasks such as tackling the SARS outbreak and strengthening preparedness for pandemic influenza, WHO has demonstrated itself to have no rival in the international health community. The International Health Regulations, revised in 2005, promise to further enhance WHO's lead role in responding to public health emergencies of international concern. Thus, what can WHO do that others cannot? What might be offered, for example, is a more coherent approach to health development at the country level, informed by global level strategies and supported by coordinated technical expertise and mobilization of support through its unrivalled networks. Already, an increasing number of developing country governments are calling on WHO to act as a broker and neutral coordinator. Many donor governments are also calling for 'one UN' country teams. $^{7}$

Recognizing the need to adapt to a more crowded health policy arena, WHO has reached out to form an unprecedented range of 'partnerships'. In some cases, engagement with so-called non-state actors (private sector and civil society groups) has proven effective. The successful negotiation of the Framework Convention on Tobacco Control was much facilitated by a huge number of groups representing the tobacco control community. More controversial has been WHO's engagement with the pharmaceutical industry as major users of knowledge from WHO, a source of donations, and research and development capacity. Against a backdrop of intense debates over patent rights and access to drugs, WHO has defended its openness to public-private partnerships on the grounds of necessity and economic pragmatism. ${ }^{8}$ However, critics worry that WHO risks compromising its responsibility as the highest global health policy setting body and the fundamental values and goals which underpin this role. Can profit-making commercial enterprises, for example, contribute to the goals of equity and social justice which underpin primary health care? Potential conflicts of interest arising from the marketing practices of some pharmaceutical companies, or their role in drafting clinical guidelines or defining specific medical conditions, ${ }^{9}$ also raise questions about how independent WHO must remain from industry. While conflict of interest guidelines have been implemented within the organization, many continue to question their transparency and effectiveness. There are also concerns that close links with industry might adversely influence other funders or discourage them from supporting the organization. And some have accused WHO of internal censorship when it comes to criticism of the pharmaceutical industry. ${ }^{10}$ The new Director-General will need to reflect critically on how to balance the value of such partnerships with concerns about the organization's integrity and independence from commercial influence.

Perhaps the most immediate task greeting the new Director-General will arise from the election process itself. 
While ostensibly intended to find the best person for the job, the reality is that the election is a hard fought battle for power and influence, not only within WHO but the international community itself. The ongoing reform of the wider UN system, alongside the appointment of the next Secretary General, provides added uncertainty. Once candidates are named, old fashioned politicking commences behind closed doors. Some discussions will centre on issues concerning leadership quality; much will be about horse trading with non-health issues. History has shown that certain members of the Executive Board can exert substantial influence over the selection of the DirectorGeneral, and therefore the direction that the organization ultimately takes.

Amid this flexing of political and economic muscle, invariably the election process can divide member states by region, wealth and ideology. When all is said and done, and the new Director-General is safely ensconced in office, member states must then be brought back together behind a coherent vision. WHO's structure does little to facilitate this. Created as an amalgamation of existing and newly created regional health organizations of varying needs, resources and efficacy, WHO has been described more as several fiefdoms. Regional directors are elected by the member states of their regional offices and thus enjoy an independent base of political support. Previous DirectorGenerals have grappled with the independence of the regional offices with varying degrees of failure. Adding to this lack of internal cohesion is the scarcity of funding for WHO programmes which breeds internal competition. This chronic lack of funding, accompanied by the rise of EBFs, has meant layoffs or insecure short term contracts for a substantial proportion of WHO's staff. The death of Lee Jong-wook has clearly dampened staff morale at a time when the organisation needs to find inner strength.

How effectively the above challenges, emanating from both within and outside of WHO, are juggled during the election process and by the new Director-General will determine whether the organization can reassert a lead role in global health. It is clear that the incumbent will need to be a consummate multi tasker. Reconciliation of the multitude of interests, values and aspirations of the diverse constituencies that make up the fiercely diverse global health community is unlikely to be an option. Instead, leadership will mean articulating a clear vision and core values that will inspire many. It may even mean challenging powerful vested interests inside and outside of the organization. It will not be a job for the faint hearted.

There is no shortage of sceptics doubting WHO's ability to get its own house in order, let alone take up the leadership mantle. Yet, curiously perhaps, there remain an even greater number of supporters keen to see WHO succeed. Why? Because, despite all of its shortcomings, the world needs WHO. It is indeed ironic that, as WHO struggles to keep pace with rapid globalization, the globalizing world in which we live increasingly needs WHO. There is no other organization that can combine the necessary technical and moral authority to tackle the critical health needs of an increasingly interconnected world. Members of WHO's Executive Board, who choose the individual to be put forward for approval by the World Health Assembly as the new Director-General, would do well to keep clear in their minds a vision of what the organization could be. Beginning to achieve this vision would mean resisting dominance of proceedings by powerful governments bent on asserting their right to take their turn at the helm. It would mean assessing candidates on their merits and not their ability to placate vested interests. It would mean allocating a level of resources to WHO that matches the expectations heaped upon it. It is only with concerted support that the organization will begin to prove the sceptics wrong.

\section{REFERENCES}

1 WHO. Engaging for Health, Eleventh General Programme of Work, A Global Health Agenda. Geneva: World Health Organization.

2 Monitoring the 11th General Programme of Work, Addendum for the World Health Assembly, May 2006.

3 Vaughan JP, Mogedal S, Kruse SE, Lee K, Walt G, de Wilde K. Financing the World Health Organization: global importance of extrabudgetary funds. Health Policy 1996;35;March:229-45.

4 Kim JY, Ammann A. Is the ' 3 by 5' Initiative the Best Approach to Tackling the HIV Pandemic? PLoS Medicine 2004; 1(2): e97-e100. http: / / medicine.plosjournals.org/archive/1549-1676/1/2/pdf/ 10.1371_journal.pmed.0010037-L.pdf

5 Prah Ruger J, Yach D. Global functions at the World Health Organization. BMJ 2005;330;14 May:1099-1100.

6 Buse K, Walt G. Global public-private partnerships: Part I-a new development in health? Bulletin of the World Health Organisation 2000; 78(4):549-561.

7 High-level Panel on System-wide Coherence. Terms of Reference for New Study on United Nations System-Wide Coherence in the Areas of Development, Humanitarian Assistance, and the Environment. New York, USA: United Nations Secretariat, 2006. Available at http://www.un.org/events/ panel/html/page2.html (accessed 15 September 2006)

8 Motchane JL. Health for All or Riches for Some? WHO's responsible? Le Monde Diplomatique 2002; July. http://mondediplo.com/2002/07/ 17who (accessed 24 August 2006)

9 Moynihan R, Henry D. The fight against disease mongering: generating knowledge for action. PLoS Medicine 2006;3(4):425-28.

10 Hayes L. Industry's Growing Influence at the WHO. Health Action International, Global Policy Forum, 15 February 2001. Available at http: / / www.globalpolicy.org/reform/2001/0223who.htm (accessed 6 September 2006) 